\title{
A VIP-like peptide co-occurs with substance $P$ and enkephalin in cholinergic preganglionic terminals of the avian ciliary ganglion
}

\author{
Anton Reiner \\ Department of Anatomy and Cell Biology, The University of Michigan, Ann Arbor, MI 48109 (U.S.A.)
}

(Received 22 September 1986; Revised version received 20 January 1987; Accepted 9 March 1987)

Key words: Vasoactive intestinal polypeptide (VIP); Ciliary ganglion; Substance P; Enkephalin; Co-existence; Cholinergic; Parasympathetic; Pigeon

Using single-label and double-label immunohistochemical techniques, many of the substance P-enkephalin containing preganglionic terminals of the avian ciliary ganglion were found to also contain a VIPlike peptide. Since these terminals are also known to be cholinergic, these results suggest that neurotransmission at many synapses of the avian ciliary ganglion involves 3 different neuropeptides and a conventional neurotransmitter, acetylcholine.

The ciliary ganglion is an intraorbital parasympathetic ganglion that gives rise to postganglionic fibers that innervate the iris and ciliary body, thereby controlling pupil constriction and accommodation [14, 15, 21]. In birds, the ciliary ganglion is known to also innervate the choroidal vasculature $[14,15]$. The ciliary ganglion receives its preganglionic fibers from the visceral subdivision of the oculomotor nuclear complex, i.e. the nucleus of Edinger-Westphal (EW) [4, 12]. In birds, preganglionic terminals on ciliary ganglion neurons that innervate the choroid (termed choroidal neurons) end as boutons that encircle the target neuron, while preganglionic terminals on neurons that innervate the iris and ciliary body (known as ciliary neurons) end as a calyx or cap. Each ciliary neuron only receives a single cap-like ending, while each choroidal neuron is contacted by numerous boutonal endings. Studies in birds have shown that transmission between preganglionic fibers to the ciliary ganglion and neurons of the ciliary ganglion is cholinergic [10, 14]. Recently, substance $P(S P)$ and enkephalin (ENK) have been found to co-occur in a large number of the preganglionic endings in the avian ciliary ganglion $[3,5,26]$. Such co-occurrence was observed both in endings on choroidal neurons and in endings on ciliary neurons. Receptor binding studies and physiological studies indicate that both SP

Correspondence: A. Reiner, Department of Anatomy and Cell Biology, The University of Michigan, Ann Arbor, MI 48109, U.S.A. 

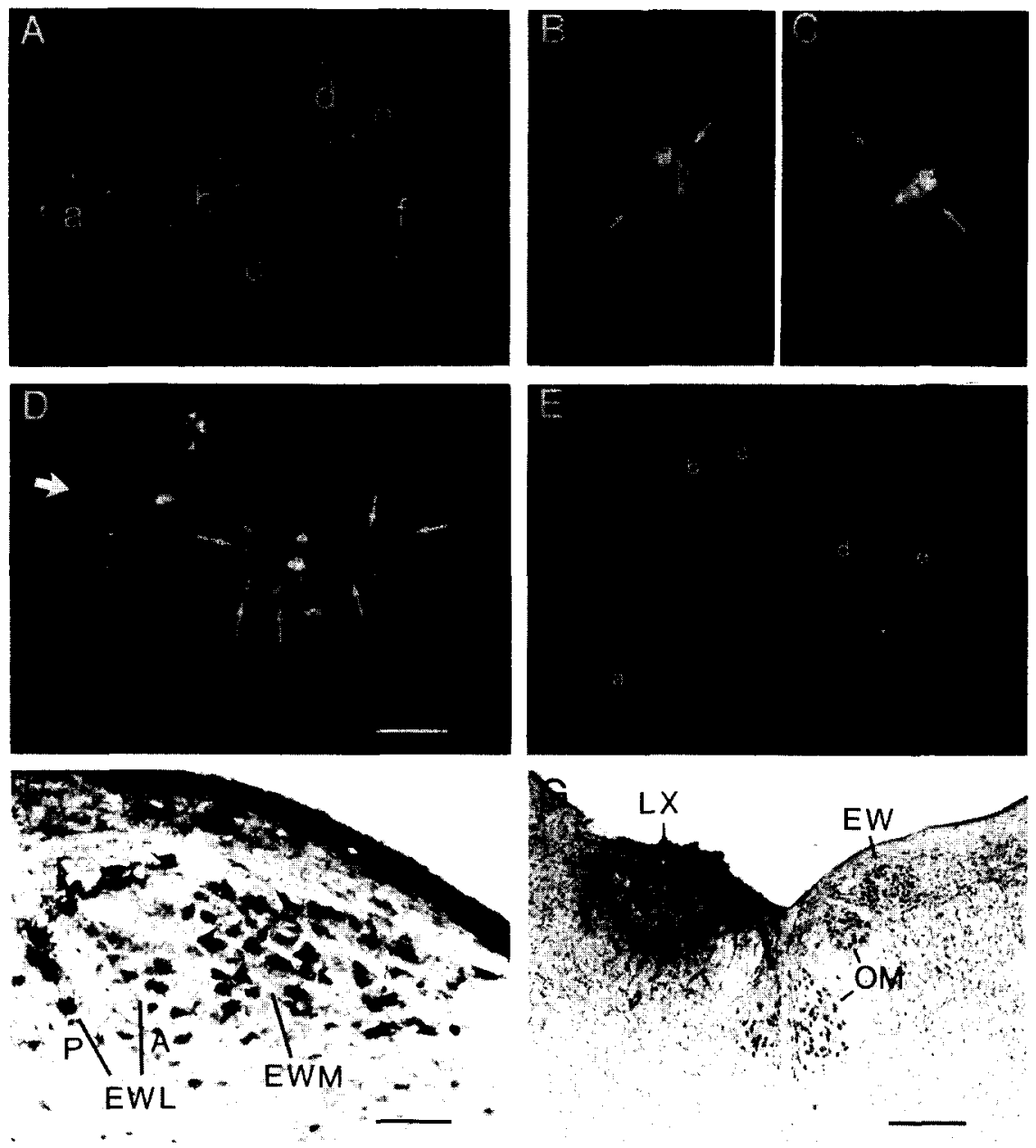

Fig. 1. A: VIP + boutonal endings on each of 6 choroidal neurons (labeled a- $f$ ). For each of the choroidal neurons, some of the individual labeled boutonal endings are indicated by small arrows. The choroidal neurons shown are $20-25 \mu \mathrm{m}$ in size. B, C: the VIP + cap-like ending (indicated by the long arrow) on each of two ciliary neurons (indicated by the short arrow). Ciliary neurons are $30-40 \mu \mathrm{m}$ in size. D: the VIP + perikarya (some of which are indicated by the medium-sized arrows) in the pterygopalatine ganglion of pigeon. The large arrow indicates a blood vessel, on which some VIP + nerve fibers (small arrows) are present. $\mathrm{Bar}=100 \mu \mathrm{m}$. E: the preganglionic endings on neurons in the ciliary ganglion are not labeled when the anti-VIP antiserum is blocked with $50 \mu \mathrm{M}$ synthetic VIP. Some of the neurons in this field of view are indicated by the letters a-f. F: VIP + neurons in EW labeled according to the peroxidase antiperoxidase procedure. Numerous labeled neurons are present in medial EW (EWM), which gives rise to the boutonal endings. Lateral EW (EWL) gives rise to the cap-like endings and EWL consists of two subdivisions [21], one controlling pupil diameter (P) and one controlling accommodation (A). VIP + neurons are abundant and intensely labeled in the portion of EWL that controls pupil diameter, but less abundant and intensely labeled in the accommodative subdivision of EWL. Bar $=100 \mu \mathrm{m}$. G: the extent of the lesion of EW (LX) in one of the birds sustaining complete destruction of EW. The location of the somatic subdivisions of the oculomotor nuclear complex $(\mathrm{OM})$ is indicated. Complete destruction of EW eliminated all VIP-, all SP- and all ENK-containing preganglionic fibers from the ipsilateral ciliary ganglion, with no effect on these types of terminals in the contralateral ciliary ganglion. Bar $=500 \mu \mathrm{m}$. 

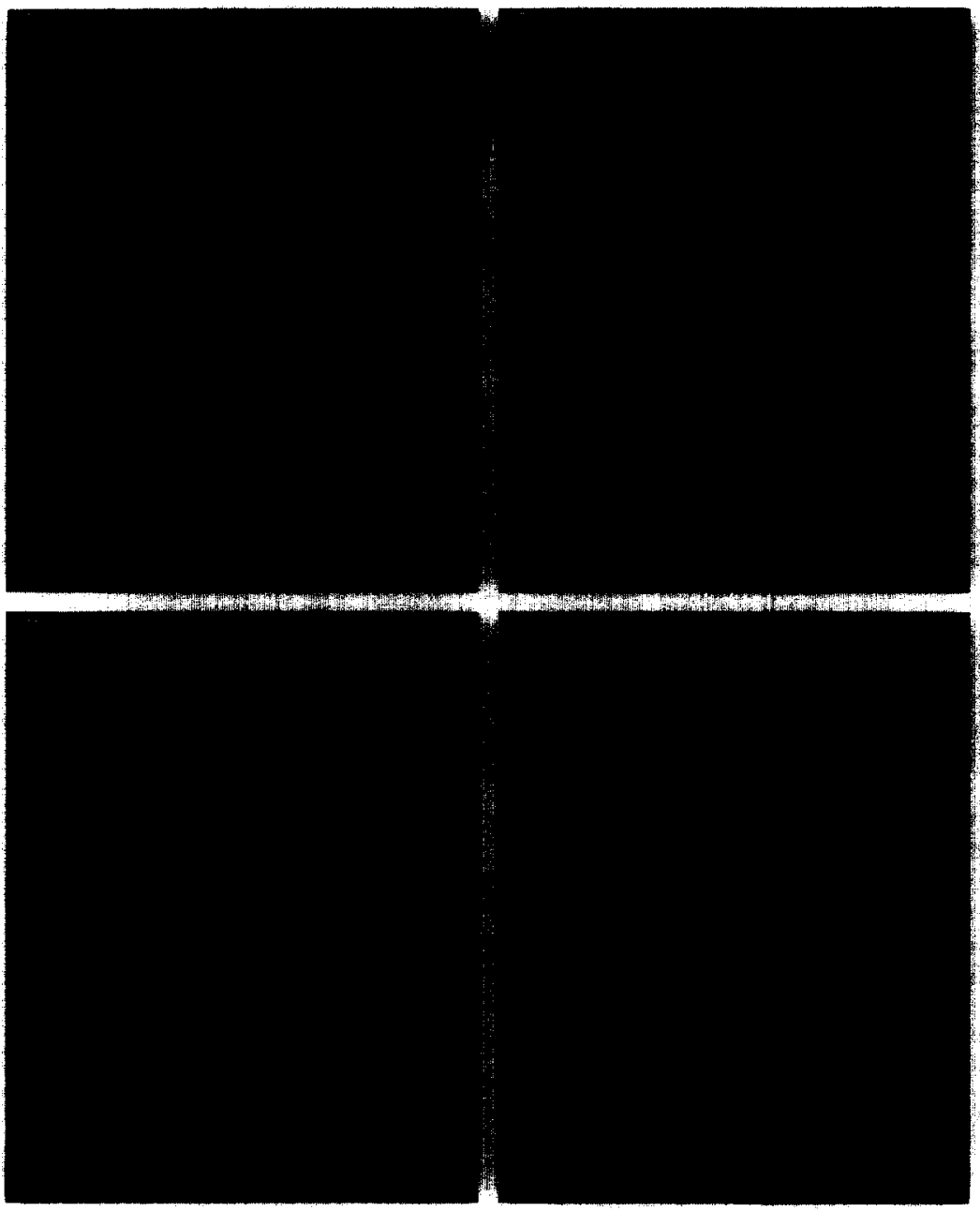

Fig. 2. A: the VIP + labeling in a section through the ciliary ganglion that had been double-labeled for VIP and SP. FITC-labeled VIP + boutonal endings are present on many of the choroidal neurons in this field of view. Eight of these choroidal neurons are identified by the letters a-i. Some of the VIP + terminals on each of these choroidal neurons are indicated by the arrows. Additional boutonal endings not indicated by arrows are also present. B: taken of the same field of view as shown in A, using the filter combination for viewing TRITC-labeling for SP. The same 8 neurons as identified by letters $a-\cdots$ in $A$ are identified by these letters in $\mathrm{B}$. As can be seen for the individual choroidal neurons shown in both photomicrographs, the terminals labeled for VIP in A are also labeled for SP in B. For example, the VIP + terminals on neuron a that are indicated by arrows in $\mathrm{A}$ are also $\mathrm{SP}+$ in $\mathrm{B}$, as shown by the arrows in $\mathrm{B}$. It should be noted that not all double-labeled boutonal endings in $\mathrm{A}$ and $\mathrm{B}$ have been indicated by arrows and not all choroidal neurons whose preganglionic endings are labeled for both VIP and SP have been indicated by letters. $\mathrm{C}$ : the VIP + labeling in a section through the ciliary ganglion that had been double-labeled for VIP and ENK. FITC-labeled VIP + boutonal endings are present on many of the choroidal neurons in this field of view. Four of these choroidal neurons are identified by the letters a-d. Some of the VIP + terminals on each of these choroidal neurons are indicated by the small arrows. Additional boutonal endings not indicated by small arrows are also present. The neuron designated by the letter $\mathrm{e}$ is a ciliary neuron (short medium-sized arrow) that receives a VIP + cap-like ending (long medium-sized arrow). Not all of this 
and ENK play neuroactive roles in the ciliary ganglion [1, 2, 6, 13, 23]. Since SP and ENK co-occur in cholinergic preganglionic terminals, individual preganglionic terminals, thus, appear to be able to influence the activity of postganglionic neurons by means of one conventional transmitter and at least two neuropeptides. In the present paper, it is reported that a VIP-like peptide is also present in many preganglionic terminals of the avian ciliary ganglion and co-occurs with SP and ENK.

Ciliary ganglia were dissected from 6-9-month-old pigeons that had been transcardially perfused with $6 \%$ dextran in $0.1 \mathrm{M}$ phosphate buffer $(\mathrm{pH} 7.2)$ followed by 100 $200 \mathrm{ml}$ of the paraformaldehyde lysine-periodate fixative as described previously $[19,22]$ and then stored in $30 \%$ sucrose $-0.02 \%$ sodium azide $0.1 \mathrm{M}$ phosphate buffer until sectioned on a cryostat $[3,5]$. Using immunofluorescence techniques as described previously $[19,22], 20-\mu \mathrm{m}$ cryostat sections through the pigeon ciliary ganglion were examined using a 1:1000 dilution of an anti-VIP antiserum (obtained from ImmunoNuclear Corporation). Antisera were applied as droplets to the slide mounted sections. Numerous VIP + boutonal endings were observed on the majority of choroidal neurons (Figs. 1 A and 2A,C) and VIP + cap-like endings were observed on many of the ciliary neurons (Fig. 1B, C). No VIP + labeling was observed, however, in neurons of the ciliary ganglion. The absence of VIP + labeling in ciliary ganglion neurons did not appear to be due to an insensitivity of the present immunohistochemical procedures to peripheral VIP-containing cell bodies. Numerous perikarya that were intensely labeled for VIP were observed in cryostat sections of the pterygopalatine ganglion (Fig. 1D), as has been previously observed by Walcott et al. [24]. All VIP + labeling could be blocked by including $50 \mu \mathrm{M}$ synthetic VIP in the primary antiserum incubation medium (Fig. 1E).

Since SP and ENK have been previously found to co-occur in boutonal endings on the vast majority of choroidal neurons and in cap-like endings on many of the ciliary neurons $[4,5]$, double-labeling procedures were used to determine if VIP was present in the same endings that contain SP and ENK. In these double-label studies, simultaneous immunofluorescence double-labeling procedures were used, as described previously [5, 18, 20, 25], using a Leitz epi-illumination fluorescence microscopy system. Control procedures were carried out as described previously $[5,18,20$. 25] to ensure that the double-labeling observed was not the spurious consequence of unintended antisera cross-reactivity or of fluorophore cross-emission. To study the

cap-like ending is present in this section; and therefore, the cap-like ending does not form a continuous cap as scen in Fig. IB, C. D: taken of the same field of view as shown in C, using the filter combination for viewing TRITC-labeling for ENK. The same 5 neurons as identified by letters a $\mathrm{e}$ in $\mathrm{C}$ are identified by these letters in $\mathrm{D}$. As can be seen for the individual neurons shown in both photomicrographs, the terminals labeled for VIP in C are also labeled for ENK in D. For example, the VIP + terminals on neuron a that are indicated by arrows in $C$ are also ENK + in D, as shown by the arrows in $D$. Not all doublelabeled boutonal endings in $C$ and $D$ have been indicated by arrows and not all choroidal neurons whose preganglionic endings are labeled for both VIP and ENK have been indicated by letters. Finally, as noted in the text and as can be seen in the two pairs of above photomicrographs, a minority of the choroidal neurons receive SP + terminals that do not contain VIP + labeling and a minority of the choroidal neurons receive ENK + terminals that do not contain VIP labeling. 
co-localization of VIP and SP, the anti-VIP antiserum (which was raised in rabbit) was used in conjunction with an FITC-labeled anti-rabbit IgG secondary antiserum (raised in goat), and a rat monoclonal antibody against SP (Accurate Chemical and Scientific Co.) was used in conjunction with a TRITC-labeled anti-rat IgG secondary antiserum (raised in goat). Using these primary and secondary antisera according to the simultaneous immunofluorescence procedure, the tissue was simultaneously FITC-labeled for the presence of VIP and TRITC-labeled for the presence of SP. Individual labeled fibers and terminals were visualized using fluorescence microscopy and examined, with the appropriate filter combinations, to see if they were both FITC-labeled and TRITC-labeled. A similar approach was used to determine if ENK and VIP were co-localized in preganglionic terminals in the ciliary ganglion. To colocalize ENK and VIP, the VIP antiserum was used in conjunction with the FITClabeled anti-rabbit IgG secondary antiserum (raised in goat), and a mouse monoclonal antibody against leucine-enkephalin (Accurate Chemical and Scientific Co.) was used in conjunction with a TRITC-conjugated anti-mouse IgG secondary antiserum (raised in goat). Using this double-labeling approach, it was found that the VIP + boutonal endings on nearly all of the choroidal neurons (96\%) that were contacted by VIP + boutonal endings contained both VIP and SP (Fig. 2A, B). Further, the VIP + boutonal endings on nearly all of the choroidal neurons $(94 \%)$ contacted by VIP + boutonal endings contained both VIP and ENK (Fig. 2C, D). The boutonal endings on only a minority of the neurons contacted by SP + boutonal endings (20\%) and on only a minority of the neurons contacted by ENK + boutonal endings (20\%) did not contain VIP. Since SP and ENK have been found to co-occur in nearly all boutonal endings in the ciliary ganglion [5], the present results indicate that the majority of the boutonal endings in the ciliary ganglion contain VIP, SP and ENK. In the case of cap-like endings, nearly all VIP+ endings were observed to contain SP (95\%) as well as ENK (99\%). Thus, it seems evident that VIP, SP and ENK must all co-occur in many cap-like endings. A number of the SP+ cap-like endings (60\%), however, were found that did not contain observable VIP. Similarly, many ENK + cap-like endings did not contain observable VIP (40\%). Thus, VIP may only be present in a subpopulation of the cap-like endings.

The source of the SP/ENK-containing terminals in the ciliary ganglion has been shown to be $\mathrm{EW}[4,12]$. Since VIP was found almost exclusively in these peptidergic terminals, it was concluded that neurons of EW must also contain VIP. In order to demonstrate this, sections through $\mathrm{EW}$ from colchicine-treated pigeons $[18,22]$ were examined for the presence of VIP + neurons using immunohistochemical procedures. Up to $45 \%$ of the neurons in medial EW and $25 \%$ in lateral EW were observed to contain VIP (Fig. 1F). The relative abundance of VIP + neurons in the two subdivisions of EW and the relative abundance of the two types of VIP + terminals in the ciliary ganglion are concordant with previous suggestions that medial EW is the source of boutonal endings and lateral EW the source of cap-like endings [7, 8, 21]. Consistent with the results of the double-label studies on the ciliary ganglion, doublelabel studies on sections through EW showed that VIP + neurons in EW also contained SP and ENK. To confirm that EW was the source of the VIP + endings in the ciliary ganglion, EW was lesioned electrolytically $(2 \mathrm{~mA}$ anodal current for 30 s), using stereotaxic procedures, in several pigeons (Fig. 1G). After 10-14 days, the ciliarv ganolia incilateral and contralateral to the lesions were examined usino immu- 
nohistochemistry. After total EW lesions, as confirmed histologically, all VIP + preganglionic fibers and terminals were eliminated from the ipsilateral ciliary ganglion. In the contralateral ciliary ganglion, the VIP + fibers were normal in appearance and number.

The present results indicate that a VIP-like substance is present in cholinergic parasympathetic preganglionic fibers and terminals in the avian ciliary ganglion. Presumably this substance is the avian homologue of mammalian VIP. Previous studies have reported that VIP is present in neurons of several other well-studied cholinergic systems [11], and a neuroactive role for VIP has been demonstrated in some of these. The VIP + fibers in the ciliary ganglion arise from VIP + neurons of EW. The majority of the choroidal neurons of the ciliary ganglion are innervated by fibers that clearly contain SP and ENK as well as VIP. Since these preganglionic endings are cholinergic, the present results suggest that the majority of the boutonal endings may influence choroidal neurons by means of at least 3 neuropeptides (SP, ENK and VIP) and a conventional neurotransmitter (acetylcholine). VIP was also observed in the present study in cap-like endings on ciliary neurons. Fewer cap-like endings than boutonal endings, however, were observed to contain VIP. It is possible, nonetheless, that many cap-like endings (as well as some boutonal endings) contained levels of VIP that were below the detectable level for the present study. Since the cap-like endings are also known to be cholinergic $[10,14]$ and since the vast majority of the $\mathrm{VIP}+$ cap-like endings were observed to contain SP and ENK, the present results indicate that all 3 neuropeptides, as well as acetylcholine, are present in many individual cap-like endings. In vitro electrophysiological studies of intact ciliary ganglion or dissociated ciliary ganglion neurons have shown that SP and ENK clearly have effects on both types of ciliary ganglion neurons [1, 2, 13, 23]. Although it seems likely that VIP also can influence ciliary ganglion neurons, the effect of VIP on these neurons is currently unknown. It is also unknown how these different neuroactive substances may complement each other in their actions. Finally, it is unknown whether all or merely some of the above neuroactive substances are co-released. It is possible that only some are co-released and that the specific substances co-released may vary with the firing frequency of the preganglionic fibers $[16,17]$. Despite the uncertainty about the role of VIP in the ciliary ganglion, the present results suggest that neurotransmission within the ciliary ganglion may be even more complex than recently thought; individual terminals in the ciliary ganglion appear capable of affecting postsynaptic responses by releasing any or all of 3 different neuropeptides and one conventional transmitter.

Special thanks are in order to Gary Henderson for technical assistance and to Lynn Cutler for secretarial assistance. This research was supported by NS-19620 and EY -05298.

I Dryer. S.E. and Chiappinelli, V.A., Electrophysiological evidence for substance $P$ as a neurotransmitter in the ciliary ganglion, Soc. Neurosci. Abstr., 11 (1985) 707.

2 Dryer, S.E. and Chiappinelli, V.A., Properties of choroid and ciliary neurons in the avian ciliary ganglion and cvidence for substance $P$ as a neurotransmitter, J. Neurosci., 5 (1985) 26542661

3 Erichsen, J.T., Karten, H.J., Eldred, W.D, and Brecha, N.C., Localization of substance P-like and enkephalin-like immunoreactivity within preganglionic terminals of the avian ciliary ganglion: light and electron microscopy. J. Neurosci., 2 (1982) 994-1003. 
4 Erichsen, J.T., Reiner, A., Cabot, J.B. and Karten, H.J., Neurons of the nucleus of Edinger-Westphal are the source of enkephalinergic and substance P-containing terminals in the avian ciliary ganglion. Soc. Neurosci. Abstr., 7 (1981) 777.

5 Erichsen, J.T., Reiner, A. and Karten. H.J., The co-occurrence of substance P-like and leucine-enkephalin-like immunoreactivities in neurons and fibers of the avian nervous system, Nature (London). 295 (1982) 407-410

6 Erichsen, J.T., Keyser, K.T., Zukin, R.S. and Karten, H.J., Opiate receptors: characterization in the avian ciliary ganglion, Soc. Neurosci. Abstr., 10 (1984) 989.

7 Gamlin, P.D.R., Reiner, A., Erichsen, J.T., Cohen, D.H. and Karten, H.J., The neural substrate for the pupillary light reflex in pigeons, $J$. Comp. Neurol., 226 (1984) $523-543$.

8 Gamlin, P.D.R., Reiner, A. and Karten, H.J., Substance P-containing neurons of the avian suprachiasmatic nucleus project directly to the nucleus of Edinger-Westphal, Proc. Natl. Acad. Sci. USA, 79 (1982) $3891 \% 3895$

9 Hess, A., Pilar, G. and Weakly, J.N., Correlation between transmission and structure in avian ciliary ganglion synapses, J. Physiol. (London), 202 (1969) 339-354.

10 Landmesser, L. and Pilar, G., The onset and development of transmission in the chick ciliary ganglion. J. Physiol. (London), 222 (1972) 691-713.

11 Lundberg, J.M., Anggard, J.M. and Fahrenkrug, J., Complementary role of vasoactive intestinal polypeptide (VIP) and acetylcholine for cat submandibular gland blood flow and secretion. III. Effects of local infusions, Acta Physiol. Scand., 114 (1982) 329-337.

12 Lyman, D. and Mugnaini, E., The avian accessory oculomotor nucleus. Soc. Neurosci. Abstr., 6 (1980) 479.

13 Margiotta, J.F. and Berg, D.K., Enkephalin and substance P modulate synaptic properties of chick ciliary ganglion neurons in cell culture, Neuroscience, 18 (1986) 175182.

14 Martin. A.R. and Pilar, G., Dual mode of synaptic transmission in the avian ciliary ganglion, J. Physiol. (London), 168 (1963) 443463.

15 Marwitt, R., Pilar, G. and Weakly, J.N., Characterization of two ganglion cell populations in avian ciliary ganglion, Brain Res., 25 (1971) 317-334.

16 Philippe. E. and Tremblay, J.P.. In vivo stimulation of a cholinergic synapse of the chick ciliary ganglion induces a reduction in the number of dense core vesicles, Neurosci. Lett., 24 (1981) $307 \quad 312$.

17 Philippe, E. and Tremblay, J.P., Increased number per area of peptidergic and cholinergic vesicles in synapses of the chick ciliary ganglion following $10 \mathrm{~Hz}$ in vivo stimulation, Neurosci. Lett., 35 (1983) 149 I 54.

18 Reiner, A.. The co-occurrence of substance P-like immunoreactivity and dynorphin-like immunoreactivity in striatopallidal and striatonigral projection neurons in birds and reptiles, Brain Res., 371 (1986) 155161

19 Reiner, A., Davis, B.M., Brecha, N.C. and Karten, H.J., The distribution of enkephalin-like immunoreactivity in the telencephalon of the adult and developing domestic chicken, J. Comp. Neurol., 228 (1984) $245 \cdots 262$.

20 Reiner, A., Eldred, W.D., Beinfeld, M.C. and Krause, J.E., The co-occurrence of a substance P-like peptide and cholecystokinin-8 in a fiber system of turtle cortex, J. Neurosci., 5 (1985) 15221526.

21 Reiner, A., Karten, H.J., Gamlin, P.D.R. and Erichsen, J.T., Parasympathetic control of ocular function: functional subdivisions and connections of the avian nucleus of Edinger-Westphal, Trends Neurosci.. 6 (1983) 140-145.

22 Reiner, A., Karten, H.J. and Solina, A.R., Substance P: localization within paleostriatal-tegmental pathways in pigeons, Neuroscience, 9 (1983) 61-85.

23 Role, L.W., Substance P modulation of acetylcholine-induced currents in embryonic chicken sympathetic and ciliary ganglion neurons, Proc. Natl. Acad. Sci. USA, 81 (1984) 29242928.

24 Walcott, B., Keyser, K.T., Sibony, P. and Karten, H.J., The histochemistry of the pterygopalatine ganglion and harderian gland of the chicken, Soc. Neurosci. Abstr., 10 (1984) 434.

25 Wessendorf, M. and Elde, R.P., Characterization of an immunofluorescence technique for the demonstration of co-existing neurotransmitters within nerve fibers and terminals, J. Histochem. Cytochem., 33 (1985) 984 994.

26 White, J.D., Krause, J.E., Karten, H.J. and McKelvy, J.F., Presence and ontogeny of enkephalin and substance $P$ in the chick ciliary ganglion, J. Neurochem., 45 (1985) 1319--1322. 